\title{
Focus and Higher-Order Unification
}

\author{
Claire Gardent \\ Computational Linguistics \\ Universität des Saarlandes, \\ D-66041 Saarbrücken \\ claire@coli.uni-sb.de
}

\author{
Michael Kohlhase \\ Computer Science \\ Universität des Saarlandes, \\ D-66041 Saarbrücken \\ kohlhase@cs.uni-sb.de
}

\begin{abstract}
Pulman has shown that Higher-Order Unification (HOU) can be used to model the interpretation of focus. In this paper, we extend the unification-based approach to cases which are often seen as a test-bed for focus theory: utterances with multiple focus operators and second occurrence expressions. We then show that the resulting analysis favourably compares with two prominent theories of focus (namely, Rooth's Alternative Semantics and Krifka's Structured Meanings theory) in that it correctly generates interpretations which these alternative theories cannot yield. Finally, we discuss the formal properties of the approach and argue that even though HOU need not terminate, for the class of unification-problems dealt with in this paper, HOU avoids this shortcoming and is in fact computationally tractable.
\end{abstract}

\section{Introduction}

In this paper, we argue that Higher-Order Unification (HOU) provides a linguistically adequate tool for modeling the semantics of focus. Building up on (Pulman, 1995), we develop a unificationbased analysis of focus which we show favourably compares with two prominent theories of focus, Rooth's Alternative Semantics and Krifka's Structured Meanings theory. For data which is generally viewed as a test-bed for focus theory (utterances with multiple focus operators and second occurrence expressions), we show that contrary to Rooth's and Krifka's theories, the HOU treatment yields a transparent analysis while avoiding under- and over-generation.

\section{Focus theory}

Focus is a much debated notion. In this paper, we assume a simplified version of Jackendoff's definition: a focus is the semantic value of a prosodically prominent element. We take the identification of prosodically prominent elements as given.

To set the stage for this paper, we will briefly review the folklore, i.e. the main issues of focus theory. It is commonly agreed that focus triggers the formation of an additional semantic value which we will call the Focus Semantic Value (FSV). The name and definition of the FSV varies from author to author: Jackendoff (Jackendoff, 1972) calls it the presuppositional set, Rooth (Rooth, 1992) the Alternative Set and Krifka (Krifka, 1992) the Ground. In this paper, we assume a definition of the FSV which is in essence Rooth's Alternative set, that is, the set of semantic objects obtained by making an appropriate substitution in the focus position. For instance, the FSV of (1a) is defined as (1b), the set of properties of the form like-ing $y$ where $y$ is an individual (in what follows, focus is indicated using upper-case; we also follow Montague's convention that for any type $\tau, D_{\tau}$ is the set of objects of type $\tau$ and $w f_{\tau}$ is the set of wffs of type $\tau$ ).

$\begin{array}{ll}\text { (1) a. Jon only likes } M A R Y \\ \text { b. } & \left\{\lambda x . l(x, y) \mid y \in D_{e}\right\}\end{array}$

It is also usually agreed that certain linguistic elements associate with focus in that the meaning of the utterance containing these elements varies depending on the choice of focus. For instance in $(2 \mathrm{a}-\mathrm{b})$, the focus operator only associates with focus so that the difference in focus between (2a) and (2b) induces a difference in meaning between the two utterances: in a world where Jon introduced Paul to Mary and Sarah, and no other introduction takes place, (2a) is necessarily 
false whilst $(2 \mathrm{~b})$ is true.

(2) a. Jon only introduced Paul to MARY

b. Jon only introduced PAUL to Mary

To model this "association-with-focus" phenomenon, the semantics of associating-elements (e.g. focus operators, quantificational adverbs) is made contingent on the FSV which itself, varies with the choice of focus. The following example illustrates this. Suppose that the meaning of only is determined by the following rule:

[NP only VP]

$\hookrightarrow \forall P\left[P \in F S V \wedge P\left(N P^{\prime}\right) \rightarrow P=V P^{\prime}\right]$

where $N P^{\prime}, V P^{\prime}$ represent the meaning of NP and VP respectively, and FSV stands for the focus semantic value of the VP. As we have seen above, the FSV of (1a) is (1b), hence by the above semantic for only, the semantics of (1a) is:

$$
\begin{gathered}
\forall P\left[P \in\left\{\lambda x . l(x, y) \mid y \in D_{e}\right\} \wedge P(j)\right. \\
\rightarrow P=\lambda x . l(x, m)]
\end{gathered}
$$

Intuitively, the only property of the form likeing $y$ that holds of Jon is the property of like-ing Mary.

\section{The basic analysis}

For computing the Focus Semantic Value, we propose to use Higher-Order Unification. More specifically, given (part of) an utterance $U$ with semantic representation $\mathrm{Sem}$ and foci $F^{1} \ldots F^{n}$, we require that the following equation, the ground equation, be solved:

$$
S e m=G d\left(F^{1}\right) \ldots\left(F^{n}\right)
$$

Assuming the typed $\lambda$-calculus as our semantic representation language, this equation can be solved by Huet's algorithm (cf. (Huet, 1975)), thus assigning a value to $G d$. On the basis of this value, we can then define the FSV, written $\overline{G d}$, as follows:

Definition 3.1 (Focus Semantic Value) Let $G d$ be of type $\alpha=\overrightarrow{\beta_{k}} \rightarrow t$ and $n$ be the number of foci $(n \leq k)$, then the Focus Semantic Value derivable from $G d$, written $\overline{G d}$, is $\left\{G d\left(t^{1} \ldots t^{n}\right) \mid\right.$ $\left.t^{i} \in w f f_{\beta_{i}}\right\}$.

As mentioned before, this yields a focus semantic value which is in essence Rooth's Alternative Set卬.

\footnotetext{
${ }^{1}$ Though in fact, our definition is more syntactic than Rooth. In Rooth's approach, the FSV definition is purely semantic whereas in our approach the FSV is indirectly defined by solving equations and the value thus obtained (i.e. the value of $G d$ ) is a term, that is, a syntactic object. Hence, our FSV can be more accurately compared to Kratzer's presupposition skeleton,. This means that our approach inherits the advantages of Kratzer's approach (cf. (Kratzer, 1991)). In par-
}

Finally, we assume as in (Pulman, 1995), that foci are stored and discharged nondeterministically as the need arises, thus contributing to the definition of the ground equation. Furthermore, equations are set up at the level at which there are needed e.g. at the VP level in the case of a pre-verbal focus operator.

To illustrate the workings of our approach, we now run through a simple example. Consider (1a). To determine the meaning of only likes MARY, the FSV of the VP must be known. Hence the following equation must be solved:

$\lambda x . l(x, m)=G d(m)$

By HOU, the value of $G d$ is then?:

$G d=\lambda y \lambda x \cdot l(x, y)$

And by definition (3.1), the FSV is:

$\overline{G d}=\left\{\lambda x . l(x, y) \mid y \in w_{f f}\right\}$

Assuming the semantic of only given above, the semantic representation of (1a) is then:

$$
\begin{aligned}
& \forall P\left[P \in\left\{\lambda x . l(x, y) \mid y \in w f f_{e}\right\} \wedge P(j)\right. \\
& \rightarrow P=\lambda x . l(x, m)]
\end{aligned}
$$

In short, we obtain a reading similar to that of Rooth, the difference being in the way the FSV is determined: by HOU in our approach, by means of a semantic definition in Rooth's.

\section{Linguistic applications}

In this section, we show that the HOU approach favourably compares with Rooth's and Krifka's analysis in that it correctly generates interpretations which these two theories fail to yield. As we shall see, the main reason for this is that the HOU approach makes minimal assumptions about the role syntax plays in determining the FSV. In particular, it relies neither on the use of Quantifier Raising, nor on the assumption of a rule-to-rule definition of the FSV. In this way, it avoids some of the pitfalls these theories encounter.

ticular, it adequately captures the interaction of focus with VP ellipsis as illustrated by Kratzer's notorious example: I only went to TANGLEWOOD because you did.

${ }^{2}$ Unification yields another possible value of $G d$, namely $\lambda y \lambda x . l(x, m)$. In what follows, we assume a restriction similar to the DSP's Primary Occurrence Restriction (Dalrymple et al., 1991)'s: the occurrence directly associated with the focus is a primary occurrence and any solution containing a primary occurrence is discarded as linguistically invalid. For instance, $m$ is a primary occurrence in the equation $\lambda x . l(x, m)=G d(m)$ so that the solution $G d=$ $\lambda y \lambda x . l(x, m)$ is invalid. For a formal treatment of DSP's Primary Occurrence Restriction and a discussion of how it can be extended to focus, see (Gardent and Kohlhase, 1996). 
We begin by a brief summary of Rooth's and Krifka's theories and stress the properties relevant for the present discussion. We then confront the three theories with the data.

\subsection{Two alternative theories of focus}

\section{Rooth's Alternative Semantics}

In Rooth's approach, the FSV is defined by recursion on the truth-conditional structure which is itself derived from LF (i.e. Logical Form, the Government and Binding level of semantic representation). Focus is then seen as introducing a free variable whose value is determined by the current context and is furthermore constrained to be an element or a subset of the FSV. For our purpose, the following characteristics are particularly important:

- Given Rooth's definition of the Alternative Set, a focus operator associates with any focus occurring in its scope.

- Any NP may be subject to Quantifier Raising. Importantly, this includes focused NPs.

- Quantifier Raising may not apply to quantifiers occurring in a scope-island.

Note that Rooth's approach critically relies on quantifier raising as a means of moving a focused NP out of the scope of a focus operator. However this only applies if the focus NP is not embedded in a scope island.

\section{Krifka's Structured Meanings}

Krifka's approach defines a rule-to-rule semantics which assigns to any syntactic constituent, a meaning which can be either a $\lambda$-term or a structured meaning, i.e. a tuple of the form $\langle G d, F\rangle$ where $G d$ is Krifka's Focus Semantic Value and $F$ is a (possibly complex) focus.

For our purpose, an important characteristic of Krifka's approach is the tight syntax/semantic interaction it presupposes. In particular, the theory requires that a focus operator combines with a syntactic constituent $C$ whose structured semantics $C^{\prime}=\langle G d, F\rangle$ provides the focus $(F)$ this operator associates with. In other words, the rightadjacent sibling of a focus operator must contain all and only the foci this operator associates with. As we shall later see, some of the data does not seem to square with this assumption.

\subsection{Multiple Focus Operators}

Utterances with multiple focus operators are known pathological cases of focus theory:

(3) a. (Jon only read the letters that Sarah sent to $P A U L_{1}$ )

b. Jon also $o_{2}$ only 1 read the letters that $S U E_{2}$ sent to $P A U L_{1}$.

In the given context, the preferred reading of (3b) can be glossed as follows: it is also the case for $S U E_{2}$, that Jon only read the letters she sent to $P A U L_{1}$ - i.e. Jon didn't read the letters she $e_{2}$ sent to e.g. Peter. In other words, the preferred reading is that also 2 associates with $S U E_{2}$ and only $y_{1}$ with $P A U L_{1}$.

\section{The HOU analysis}

Under the HOU approach, (3b) is analysed as follows. First, the meaning of only 1 read the letters that $S U E_{2}$ sent to $P A U L_{1}$ is derived. To determine the FSV of the $\mathrm{VP}$, the ground equation (4b) must be solved for which (4c) is a solution. Applying the semantics of only given in section 2, the semantics of $(4 \mathrm{a})$ is then as given in $(4 \mathrm{~d}) \mathrm{f}$.

(4) a. only read the letters that $S U E_{2}$

$$
\text { sent to } P A U L_{1}
$$

b. $G^{1}(p)=\lambda x \cdot \operatorname{read}(x, l(s, p))$

c. $\quad G^{1}=\lambda y \cdot \lambda x \cdot \operatorname{read}(x, l(s, y))$

d. $\quad \lambda z . \forall P[P \in \overline{\lambda y \lambda x \cdot \operatorname{read}(x, l(s, y))} \wedge P(z)$

$$
\rightarrow P=\lambda x \cdot \operatorname{read}(x, l(s, p))]
$$

Analysis then proceeds further and the ground equation

$$
\begin{aligned}
G^{2}(s)=\lambda z \cdot \forall P[ & P \in \overline{\lambda y \lambda x \cdot r e a d(x, l(s, y))} \\
& \wedge P(z) \rightarrow P=\lambda x \cdot \operatorname{read}(x, l(s, p))]
\end{aligned}
$$

must be solved to determine the meaning of also $_{2}$ only $_{1}$ read the letters that $S U E_{2}$ sent to $P A U L_{1}$. A possible solution for $G^{2}$ is

$$
\begin{aligned}
\lambda u . \lambda x . \lambda z . \forall P[ & P \in \overline{\lambda y \lambda x \cdot \operatorname{read}(x, l(u, y))} \\
& \wedge P(z) \rightarrow P=\lambda x \cdot \operatorname{read}(x, l(u, p))]
\end{aligned}
$$

Assuming the following semantics for NP also VP

$$
\exists P\left[P \in F S V \wedge P\left(N P^{\prime}\right) \wedge P \neq V P^{\prime}\right]
$$

we obtain the desired reading

$$
\begin{aligned}
& \exists P\left[P \in \overline{\lambda u \lambda x . \frac{\text { only }_{1} \text { read the letters that }}{\text { u sent to } \text { Paul }_{1}}}\right. \\
& \wedge P(j) \wedge P \neq \lambda z . z \quad \text { only }_{1} \text { read the letters } \\
& \text { that } \left.\mathrm{Sue}_{2} \text { sent to } \mathrm{Paul}_{1}\right]
\end{aligned}
$$

\footnotetext{
${ }^{3}$ The subscripts indicates which operators associate with which focus. There are there for clarity only, and have no theoretical import.

${ }^{4}$ For clarity, we have simplified the semantic representation of $(3 \mathrm{~b})$; nothing hinges on this.
} 


\section{Comparison with Rooth and Krifka}

As mentioned in section 4.1, under the Alternative Semantics approach, a focus operator necessarily associates with any focus occurring in its scope. Furthermore in (3b), the scope of only is the whole VP read the letters that $S U E_{2}$ sent to $P A U L_{1}$. Hence, if no quantifier raising occurs, only $y_{1}$ associates with both $S U E_{2}$ and $P A U L_{1}$. Thus in order to generate the desired reading, $S U E_{2}$ must be moved out of the scope of only . However, since the NP the letters that $S U E_{2}$ sent to $P A U L_{1}$ is a scope island, quantifier raising is impossible. Hence, the desired reading cannot be generated

Recall that in the Structured Meanings approach, the right-sibling of a focus operator must contain all and only the focus this operator associates with (cf. section 4.1). Hence, to generate the desired reading in (3b), there must exist a syntactic constituent which is right-adjacent to only $y_{1}$ and which contains $P A U L_{1}$ but not $S U E_{2}$ [; similarly, there must exist a syntactic constituent which is right-adjacent to also and which contains $S U E_{2}$ but not $P A U L_{1}$. Given standard assumptions about syntax, such constituents do not exist so that the desired interpretation cannot be generated.

\subsection{Second Occurrence Expressions}

We call second occurrence expressions (SOE) utterances which partially or completely repeat a previous utterance. Typical cases of SOEs are: corrections (5a), echo-sentences (5b) and variants (5c).

$$
\begin{aligned}
& \text { A. Jon only likes MARY. } \\
& \text { B: No, PETER only likes Mary. } \\
& \text { b. A: Jon only likes MARY. } \\
& \text { B: Huhu, Peter only likes Mary. } \\
& \text { c. A: Jon only likes MARY. } \\
& \text { B: So what? Even PETER only } \\
& \\
&
\end{aligned}
$$

An important property of SOEs is that the repeated material is deaccented, that is, it is characterised by an important reduction in pitch, amplitude and duration (cf. (Bartels, 1995)). On the other hand, all three theories of focus considered here are based on the assumption that focus is prosodically marked and thus, identifiable. Hence,

\footnotetext{
${ }^{5}$ This point is independently noted in Rooth, 1992 )

This is a simplification: the constituent may in fact contain $S U E_{2}$ but this focused NP should already have been bound by some focus operator so that the focus of the whole constituent only includes $P A U L_{1}$. Since no focus operators occur in this constituent, it follows that such constituent does not exist.
}

the question arises of whether these theories can account for SOEs.

\section{The HOU analysis}

Our proposal is to analyse SOEs as involving a deaccented anaphor which consists of the repeated material, and is subject to the condition that its semantic representation must unify with the semantic representation of its antecedent.

This is modeled as follows. Let SSem and TSem be the semantic representation of the source (i.e. antecedent) and target (i.e. anaphoric) clause respectively, and $T P^{1} \ldots T P^{n}$, $S P^{1} \ldots S P^{n}$ be the target and source parallel elements: then the interpretation of an SOE must respect the following equations:

$$
\begin{aligned}
& A n\left(S P^{1}, \ldots, S P^{n}\right)=S S e m \\
& A n\left(T P^{1}, \ldots, T P^{n}\right)=T S e m
\end{aligned}
$$

Intuitively, these two equations require that target and source clause share a common semantics $A n$, the semantics of the deaccented anaphor.

Given this proposal, the analysis of (5a) involves three equations:

$$
\begin{gathered}
A n(j)=\forall P[P \in \overline{\lambda y \lambda x . l(x, y)} \\
\wedge P(j) \rightarrow P=\lambda x . l(x, m)] \\
A n(p)=\forall P[P \in \overline{G d} \wedge P(p) \rightarrow P=\lambda x . l(x, m)] \\
G d(F)=\lambda x . l(x, m)
\end{gathered}
$$

Since neither $G d$ nor Focus are initially given, the third equation above is untyped and cannot be solved by Huet's algorithm 8 . In that situation, we can either assume some delaying mechanism or some extension of Huet's algorithm that can cope with type variables (cf. (Dougherty, 1993; Hustadt, 1991()). Resolution of the first equation yields the following solution:

$$
\begin{array}{r}
A n=\lambda y \forall P[P \\
\wedge\left\{\lambda x . l(x, y) \mid y \in w f f_{e}\right\} \\
\wedge P(z) \rightarrow P=\lambda x . l(x, m)]
\end{array}
$$

By applying $A n$ to $p$, the left-hand side of the second equation is then determined so that the second equation becomes

$$
\begin{aligned}
& \forall P[P \in \overline{\lambda y \lambda x . l(x, y)} \wedge P(p) \rightarrow P=\lambda x . l(x, m)] \\
= & \forall P[P \in \overline{G d} \wedge P(p) \rightarrow P=\lambda x . l i k e(x, m)]
\end{aligned}
$$

and the value of $G d$ is identified as being

$$
G d=\lambda y \lambda x . l(x, y)
$$

(Note further, that the third equation can now be solved thus yielding the value $m$ for the focus $F$.) That is, the HOU approach to SOEs allows us to correctly capture that fact that an SOE can

\footnotetext{
${ }^{7}$ As in (Dalrymple et al., 1991), we take the identification of parallel elements as given - for the moment.

${ }^{8}$ Even though this is not explicitly stated, Pulman's analysis (Pulman, 1995, page 6) faces a similar problem.
} 
inherit its FSV from its source clause (by unification). In (Gardent et al., 1996), we show in more detail how the analysis accounts for the interaction of focus with anaphora and definiteness in the case of a particular instantiation of SOEs, namely corrections.

\section{Comparison with Rooth and Krifka}

Under the Alternative Semantics approach, SOEs are captured as follows. It is assumed that the quantification domain of focus operators is a variable whose value is contextually determined. In the standard case (i.e. the case where the focus is prosodically marked), this quantification domain of focus operators is usually identified with the FSV of the VP. However, in the SOE cases, the assumption is that the quantification domain of focus operators is identified with the FSV of the source clause. Thus in (5a), the quantification of only in the second clause is identified with the FSV of the preceding utterance i.e. the set of properties of the form like-ing somebody.

But now, consider the following example:

(6) a. Jon only likes MARY.

b. * No, PETER only likes Sarah.

Clearly, this dialog is ill-formed in that $(6 \mathrm{~b})$ is no appropriate correction for (6a). However, under the Alternative Semantics approach, it will not be ruled out since the FSV of (6a) provides an appropriate quantification domain for the focus operator in (6b): as required by the semantic of pre-verbal only, it is a set of properties whose elements can be identified with the VP semantic value $\lambda x . l(x, m)$. Hence although Rooth's approach captures some cases of SOEs, it does not seem to provide an adequate characterisation of the phenomena at hand.

The Structured Meanings proposal distinguishes between proper- and quasi-SOEs. ProperSOEs involve an exact repetition of some previous linguistic material, and are analysed as involving an anaphor which is constrained by the restriction that it be a segmental copy of its antecedent. For instance, the semantics of only likes Mary in (5b) is not determined by the semantics of its parts but is instead identified with the semantic value of its antecedent only likes MARY in (5a). In contrast, quasi-SOEs only involve semantic equivalence between repeating and repeated material (for instance, in a quasi-SOE a repeated element may be pronominalised). Krifka claims that quasi-SOEs have prosodically marked foci and thus do not raise any specific difficulty.

However this theory faces a number of methodological and empirical difficulties. First, it is non- compositional because the meaning of the deaccented material in proper-SOEs is solely defined by the meaning of its antecedent (rather than the meaning of its parts). Second, the prosodic data is rather unclear: the assumption that quasi-SOE contains a prosodically marked focus is a moot point (cf. (Bartels, 1995)) and if it proves to be false, the analysis fails to account for quasiSOEs. Third, it is counterintuitive in that it handles separately two classes of data (i.e. quasi- and proper-SOEs) which naturally belong together. Indeed, the HOU approach can be shown to provide a uniform treatment of quasi- and properSOEs (cf. (Gardent et al., 1996)).

\section{Formal properties of the HOU approach}

The unification problem can be stated as follows: Given two terms of a logic $\mathbf{M}$ and $\mathbf{N}$, is there a substitution, $\sigma$, of terms for variables that will make the two terms identical (i.e. $\sigma(\mathbf{M})=\sigma(\mathbf{N}))$ ?

It is well-known that for Higher-Order Logic (e.g. the typed $\lambda$-calculus) the space of solutions can be infinite and furthermore, the HOU problem is only semi-decidable so that the unification algorithm need not terminate for unsolvable problems.

Fortunately, in our case we are not interested in general unification, but we can use the fact that our formulae belong to very restricted syntactic subclasses, for which much better results are known. In particular, the fact that free variables only occur on the left hand side of our equations reduces the problem of finding solutions to higher-order matching, of which decidability has been proven for the subclass of third-order formulae (Dowek, 1992) and is conjectured for the general case. This class, (intuitively allowing only nesting functions as arguments up to depth two) covers all of our examples in this paper. For a discussion of other subclasses of formulae, where higher-order unification is computationally feasible see (Prehofer, 1994).

\section{Conclusion}

In this paper, we have argued that Higher-Order Unification provides an adequate tool for computing Focus Semantic Values. To this end, we have considered data which is viewed as a testbed for focus theory and shown that, whilst existing theories either under-generate, over-generate or are methodologically unsatisfactory, the HOU approach yields a simple and transparent analysis. There appear to be two main reasons for this. 
First, the HOU analysis makes minimal assumptions about the role syntax is called to play in determining the FSV. It is defined on a purely semantic level in the sense that unification operates on semantic representations, and relies neither on quantifier raising, nor on a rule-to-rule definition of the FSV. As we have seen, this type of approach is a plausible way to avoid undergeneration.

Second, the HOU approach permits an equational analysis which can naturally be further constrained by additional equations. The interest of such an approach was illustrated in our treatment of SOEs which we characterise as involving two phenomena: the computation of an FSV, and the resolution of a deaccented anaphor. Not only did we show that this analysis is methodologically and empirically sound, we also showed that it finds a natural realisation in the equational framework of HOU: each linguistic phenomena is characterised by some equation(s) and the equations may mutually constrain each other. For instance, in the case of SOEs, we saw that the equations characterising the deaccented anaphor help determine the unidentified FSV of the utterance containing the unmarked focus.

Clearly, our approach extends to cases of adverbial quantification. For lack of space we could not develop the theory here; let us just point out that von Fintel's criticism (von Fintel, 1995) of semantic approaches to focus, also applies to Krifka's Structured Meanings analysis, but not to the HOU approach presented here. Von Fintel points out that in certain cases of adverbial quantification, a focus operator associates with an unmarked focus and does not associate with a marked focus occurring in its scope - as should be clear from this article, this is unproblematic for our analysis.

Of course, there are still many open issues. First, how does the proposed analysis interact with quantification? Second, how does it extend to a dynamic semantics (e.g. Discourse Representation Theory)?

\section{Acknowledgments}

The work reported in this paper was funded by the Deutsche Forschungsgemeinschaft (DFG) in Sonderforschungsbereich SFB-378, Project C2 (LISA).

\section{References}

[Bartels1995] Christine Bartels. 1995. Second occurrence test. Ms.
[Dalrymple et al.1991] Mary Dalrymple, Stuart Shieber, and Fernando Pereira. 1991. Ellipsis and higher-order-unification. Linguistics and Philosophy, 14:399-452.

[Dougherty1993] Daniel Dougherty. 1993. Higherorder unification using combinators. Theoretical Computer Science B, 114(2):273-298.

[Dowek1992] Gilles Dowek. 1992. Third order matching is decidable. In Proc. $L I C S-7$, pages 2-10, IEEE.

[Gardent and Kohlhase1996] Claire Gardent and Michael Kohlhase. 1996. Higher-order coloured unification and natural language semantics. In Proc. ACL96, Santa Cruz, USA. forthcoming.

[Gardent et al.1996] Claire Gardent, Michael Kohlhase and Noor van Leusen. 1996. Corrections and higher-order unification. CLAUS report 77, University of Saarland.

[Huet1975] Gérard Huet. 1995. A unification algorithm for typed $\lambda$-calculus. Theoretical Computer Science 1, pages 27-57.

[Hustadt1991] Ulrich Hustadt. 1991. A complete transformation system for polymorphic higherorder unification. Technical Report MPI-I-91228, MPI Informatik, Saarbrücken, Germany.

[Hutter and Kohlhase1995] Dieter Hutter and Michael Kohlhase. 1995. A coloured version of the $\lambda$-calculus. SEKI-Report SR-9505, Universität des Saarlandes.

[Jackendoff1972] Ray S. Jackendoff. 1972. Semantic Interpretation in Generative Grammar. The MIT Press.

[Kratzer1991] Angelika Kratzer. 1991. The representation of focus. In Arnim van Stechow and Dieter Wunderlich, editors, Semantik: Ein internationales Handbuch der zeitgenoessischen Forschung. Berlin: Walter de Gruyter.

[Krifka1992] Manfred Krifka. 1992. A compositional semantics for multiple focus constructions. In Joachim Jacobs, editor, Informationsstruktur and Grammatik. Sonderheft 4.

[Prehofer1994] Christian Prehofer. 1994. Decidable higher-order unification problems. In Alan Bundy, editor, Proc. CADE94, LNAI, pages 635-649, Nancy, France.

[Pulman1995] Steve G. Pulman. 1995. Higherorder unification and the interpretation of focus. Paper submitted for publication.

[Rooth1992] Mats Rooth. 1992. A theory of focus interpretation. Natural Language Semantics, pages 75-116. 
[von Fintel1995] Kai von Fintel. 1995. A minimal theory of adverbial quantification. Unpublished draft Ms. MIT, Cambridge, March. 